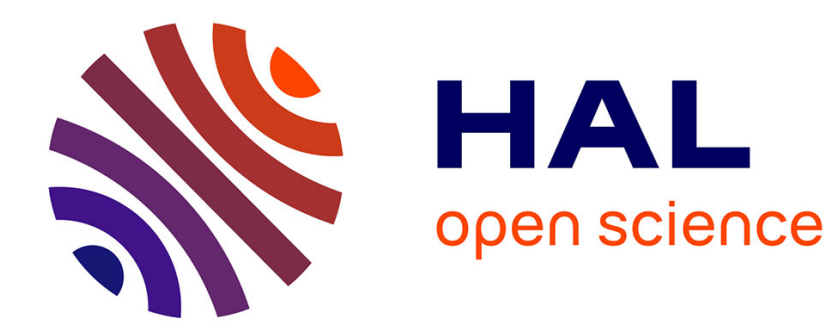

\title{
Pour une séméiologie des médias
}

François Jost

\section{- To cite this version:}

François Jost. Pour une séméiologie des médias. Signata - Annales des sémiotiques, 2011. hal01796122

\section{HAL Id: hal-01796122 \\ https://hal.science/hal-01796122}

Submitted on 19 May 2018

HAL is a multi-disciplinary open access archive for the deposit and dissemination of scientific research documents, whether they are published or not. The documents may come from teaching and research institutions in France or abroad, or from public or private research centers.
L'archive ouverte pluridisciplinaire HAL, est destinée au dépôt et à la diffusion de documents scientifiques de niveau recherche, publiés ou non, émanant des établissements d'enseignement et de recherche français ou étrangers, des laboratoires publics ou privés. 


\title{
REVUE SIGNATA 2 (2011) ANNALES DES SÉMIOTIQUES/ ANNALS OF SEMIOTICS
}

\section{La sémiotique, entre autres}

\author{
Semiotics, among others
}

Presses Universitaires de Liège - Sciences humaines 2011 



\section{THÉORIE DES MÉDIAS}

\section{Pour une séméiologie des médias}

François Jost

Sorbonne Nouvelle, Centre d'Étude des Images et des Sons Médiatiques

Depuis une vingtaine d'années que j'écris sur la télévision, j’ai souvent eu l'occasion de critiquer la sémiologie. Pourtant, s'il me faut décliner mon étiquette disciplinaire, je me définis avant tout comme un sémiologue. Cette contradiction apparente n'est pas réductible à une idiosyncrasie qui me caractériserait, elle résume à sa manière la relation complexe que la sémiologie entretient avec la théorie des médias. Complexité dont on peut donner une idée en disant qu'il est tout aussi absurde de réduire la théorie des médias à la sémiologie que de soutenir qu'elle peut s'en passer. Aussi faut-il renvoyer dos-à-dos l'impérialisme sémiotique, qui fait abstraction des conditions socio-économiques, et la sociologie des médias, plus attentive au contexte qu'aux contenus en tant que tels. Comment peut-on soutenir à la fois que la théorie de la télévision doit s'écarter de la sémiologie et revendiquer son appartenance à cette méthodologie? C'est ce que cet article va tenter d'expliquer.

\section{Ce que ne doit pas être une sémiologie des médias}

En un sens, la réponse est très simple. Elle dépend de ce qu'on va mettre sous l'étiquette " sémiologie » et du déterminant ou du complément qui va en compléter le sens. Voyons, pour commencer, ce que ne doit pas être la sémiologie si elle s'assigne la tâche metzienne de comprendre comment on comprend.

\section{Une sémiologie immanente}

Qu'on le veuille ou non, dès que l'on réfléchit sur l'image animée, les premiers travaux de Metz sont une référence obligée ou tout au moins un point de départ, même s'ils ne représentent pas un terminus ad quem. Si la fondation de la discipline 
est si importante d'un point de vue épistémologique, c'est qu'elle a fortement pesé sur ses développements ultérieurs. En effet, le pivot autour duquel tournent les Essais sur la signification au cinéma est la nécessité de donner un fondement théorique à la métaphore du «langage cinématographique ». Dès lors, s'ensuivent logiquement des recherches tournées sur le recensement et l'explicitation des codes nécessaires à la compréhension de l'image animée. De ce point de vue, "l'image cinématographique et l'image télévisuelle ne diffèrent guère que par la taille » (Metz 1971, p. 178).

À une époque où les différences de taille d'écran entre le home cinema et certaines salles de multiplexes tendent à se réduire, il apparaît évident que cinéma et télévision sont malgré tout bien différents pour des raisons qui ne tiennent pas à la seule image. L'approche metzienne peut aider à penser le medium ou les matières de l'expression, pour utiliser son vocabulaire, mais elle impuissante à analyser le média.

\section{Une sémiologie du cinéma}

Le paradoxe de cette première méthodologie, c'est qu'elle vise ostensiblement une formalisation du langage des images animées qui permet de réunir en un seul ensemble cinéma et télévision, tout en ne pensant que le cinéma. Et encore pas tout le cinéma, mais le cinéma classique de fiction. Ce terminus a quo a eu des incidences sur les premiers pas de la sémiologie de la télévision, comme on peut le constater en relisant l'un de ses textes fondateurs : «Il est là, je le vois, il me parle", d'Eliseo Véron (1983). Comme on sait, cet article montre l'importance de l'axe Y-Y, qui unit l'énonciateur et le spectateur du journal télévisé, dispositif dont il trouve l'origine dans les Actualités cinématographiques, alors même que celles-ci se distinguaient de l'actuel JT par l'absence de présentateur à l'écran. Mais peu importe. Ce qui me retient dans son raisonnement, c'est plutôt le fait qu'il fonde son analyse sur la référence au regard caméra dans le film de fiction. «Dans un film de fiction, écrit-il, le regard d'un personnage vers la caméra (lorsqu'il ne fait pas partie, bien entendu, d'un enchaînement champ/contrechamp) produit un dérèglement, une rupture majeure de la diégèse : le spectateur, plongé dans le voyeurisme complice du récit, est tout à coup surpris par le regard venant de l'image» (ibid., p. 105).

Passons sur le fait qu'un champ-contrechamp ne recourt jamais au regard à la caméra et qu'il se fonde sur le croisement des regards dans l'écran. Autrement plus gênante me semble la conséquence de ce rapprochement : l'axe Y-Y serait "une méta-opération d'identification d'un type de discours par le poids de son mouvement de défictionnalisation : une sorte de 'preuve' de l'ancrage du discours dans le réel de l'actualité » (ibid.). Le JT n’est pas une diégèse : la suite des lancements et des sujets n'est pas construite sur un monde clos sur lui-même; encore moins une fiction : même si on peut y retrouver des traits, il prétend parler de notre monde, «afilmique », comme disait Souriau (1953). On ne voit donc pas très bien le sens que peut avoir dans ces conditions une "défictionnalisation ». 
Bien qu'elle se déclare ouvertement "pragmatique », cette sémiologie reste prisonnière du cadre immanentiste de la discipline de départ et de son modèle implicite, le récit cinématographique. Car, comme l'ont montré divers travaux depuis, il est de nombreuses exceptions à l'interdiction du regard à la caméra dans les films, notamment des films muets du début $\mathrm{du} \mathrm{xx}^{\mathrm{e}}$ siècle et, plus fondamentalement, la comédie musicale américaine. Ce n'est pas un hasard si Véron note aussi l'usage télévisuel de l'axe Y-Y dans les émissions de variétés : "L'axe Y-Y produit son effet de défictionnalisation dans deux autres modalités de son apparition : les programmes de variétés et les transitions faites par les speakerines ». Au cinéma, le regard-caméra trouve son origine dans les arts de la scène : qu'il s'agisse des " attractions » du début du $\mathrm{Xx}^{\mathrm{e}}$ siècle ou des spectacles de music-hall.

Il en va tout autrement de l'axe Y-Y à la télévision. Loin d'être une « rupture majeure ", il est, pour ainsi dire l'ordinaire de la télévision, et ce depuis les origines, comme l'atteste cette recommandation de Wladimir Porché, directeur de la RTF en 1954, à tous ceux qui s'adressent au public par l'intermédiaire de la caméra " de fixer la caméra puisqu'ils s'adressent non seulement à une salle, mais à des spectateurs isolés $»^{1}$. Cette différence de construction du destinataire dit très bien le déplacement qui s'opère du cinéma à la télévision : du modèle de la scène à celui de la conversation. Si l'on est attentif à ce glissement, malgré la similarité apparente, l'axe Y-Y n'a pas du tout le même sens dans les variétés et dans les «transitions faites par la speakerine » : alors que le premier a comme interprétant le spectacle vivant et le face-à-face de l'artiste de music-hall avec le public, le second restitue une situation dialogale d'adresse, à tel point que les téléspectateurs des années 50 avaient parfois l'impression que la speakerine leur parlait ${ }^{2}$. Il arrive même en certaines occurrences que cet axe, au lieu de "défictionnaliser", soit la base de la fiction. C'est le cas de l'adaptation par Pierre Cardinal du Rouge et le Noir (1961) : qu'il s'agisse de scènes de dialogue ou de monologue, les personnages s'adressent à la caméra et, loin de produire un effet de champ-contrechamp, ce dispositif construit un tiers, qui n'est autre, évidemment, que le spectateur. Comme le dit le réalisateur à un journaliste : " les interprètes ne s'adressent pas à un partenaire : ils parlent aux seuls téléspectateurs $»^{3}$. On ne peut ancrer plus clairement le regard à la caméra au modèle de la conversation.

1. Mon programme $\mathrm{n}^{\circ} 78,17 / 4 / 1954$.

2. On trouve au détour d'un dépouillement des magazines de télévision des années 50 cette demande d'un téléspectateur à une speakerine de ne pas bouger pour qu'il puisse prendre une photo. L'écrivain Mac Orlan, qui écrit régulièrement des chroniques sur la télévision, note : « En télévision, on doit tenir compte de toutes les nuances sentimentales que les images animées provoquent chez le spectateur. Il suffit de tourner un bouton et un petit personnage très vivant, très net apparaît dans la demeure de chacun de nous. C'est un visiteur souvent inattendu. On est sur le point de lui offrir un siège, selon la plus élémentaire courtoisie. Il est donc nécessaire que ce visiteur soit sympathique [...] ce doit être un ami » (Radio 52, 22 juin 1952).

3. L'Aurore, 19 décembre 1961, cité par Delavaud (2005, p. 174) dont ce passage restitue l'analyse. 
On constate sur cet exemple combien l'origine immanentiste et cinémacentrée de la première sémiologie de l'audiovisuel pèse, y compris lorsqu'il s'agit de mettre en ouvre une visée pragmatique. L'exemple de l'axe Y-Y suggère qu'on ne peut considérer les dispositifs énonciatifs comme des codes permettant de construire une signification intangible et qu'il est préférable de les envisager comme des signes dont les interprétants varient en fonction du contexte. D'où la nécessité, pour une théorie des médias d'être intermédiale et historique.

\section{Que signifie l'adjectif « médiatique " ?}

Qu'un récit soit médiatique impose-t-il des changements majeurs dans l'arsenal conceptuel de l'analyse du récit en général?

Là encore, l'observation du cadre épistémologique fondateur de l'avènement de la narratologie et de la situation des médias aujourd'hui est instructive : alors que Genette prétendait réfléchir sur des textes, en ignorant la question de l'évaluation esthétique et artistique, l'étude du récit médiatique s'impose d'emblée comme une réflexion sur des produits ou, mieux, des biens culturels, ce qui provoque une coupure d'importance. Le système présenté par Figures III (Genette 1972), qui est le parangon de la narratologie modale, réfléchit sur des objets dont le statut est assuré : ce sont des textes littéraires de fiction, en général des « chefs-d'œuvre ». Du même coup, les lecteurs sont supposés savoir ce qu'ils lisent (au point que le statut éventuellement autobiographique d'À la Recherche du temps perdu est balayé d'une main). Nous sommes dans l'ère juridique de la théorie : lire, c'est s'engager à bien lire, et bien lire, c'est se conformer aux catégories élaborées par les théoriciens. En bref, un auteur écrit pour des lecteurs qui sont convaincus qu'ils veulent lire le livre qu'ils ont entre les mains, et qui cherchent à l'interpréter avec la bonne volonté qui sied à la plupart des schémas de la communication verbale.

Dans ce contexte, le recouvrement du récit et de la fiction sont des évidences qui vont sans dire : aussi bien chez Metz (pour qui tout récit est du côté de la fiction parce qu'il irréalise la chose racontée) que chez Genette, qui mettra dix ans à reconnaître que la narratologie ne pense que la fiction. Ce constat n'entraînera d'ailleurs chez lui aucun remaniement profond de la théorie, mais plutôt une distribution des procédés différente selon qu'on a affaire à un récit de fiction ou à un récit de réalité. Dans la lignée du Pacte autobiographique de Lejeune (1975), la narratologie genettienne ne concède qu'une ouverture vers l'auteur pour formaliser la distinction entre récit factuel et récit fictif par une égalité : $\mathrm{A}=\mathrm{N}$ et une inégalité : $\mathrm{A} \neq \mathrm{N}$.

Seuils poursuit en ce sens en recensant tous les lieux qui relient le texte à ses paratextes et autres épitextes. D’une certaine manière, toutes les théories qui envisagent la compréhension d'un texte au sens large grâce à la fiction d'un "contrat de lecture " acceptent ce rôle déterminant des "consignes» paratextuelles que sont les couvertures, les génériques ou les bandes-annonces. Et elles 
postulent du même coup que le texte fait ce qu'il dit qu'il fait. Or, précisément, ce qui distingue un objet médiatique d'un objet textuel en général, c'est qu'il ne respecte pas forcément les traits définitoires de ce qu'il prétend être. Il lui arrive de s'avancer masquer. Les exemples sont légion et je les ai suffisamment multipliés dans mes travaux pour qu'il ne soit pas utile d'y revenir lourdement. Quelques cas particulièrement notoires : Survivre avec les loups, qui a été "vendu " comme le récit autobiographique d'une femme qui, à 8 ans, a parcouru l'Europe nazie à la recherche de ses parents en compagnie de ces animaux sauvages et qui n'était qu'un roman. Les éditeurs, producteurs et diffuseurs ont une prédilection pour des objets ambigus, que l'on peut faire osciller entre le monde réel et le monde fictif. Il n'est guère de semaine sans qu'un débat s'ouvre à propos de la sortie ou du lancement de tel ou tel livre ou émission. Ce fut, il y a quelques années, le cas de la téléréalité, bien sûr, présentée à l'origine comme plus réelle que tout autre genre - au point qu'un chroniqueur célèbre put y voir le meilleur documentaire jamais fait sur la jeunesse française - ; un peu plus tard, le cas de l'Odyssée de l'espèce, téléfilm de fiction «vendu» comme un documentaire; le Da Vinci code, affiché comme roman, mais communiqué comme la révélation d'une vérité historique. Beaucoup d'autres exemples pourraient être cités tant cette ambiguïté est devenu une sorte de topos du lancement publicitaire des produits culturels.

Pour cette raison, j'ai proposé de penser la communication médiatique non comme un contrat qui lierait le producteur et le récepteur dans un acte de lecture similaire, mais comme un processus initié par une promesse. S'il revient à Genette d'avoir ouvert ce champ des seuils des ouvrages, il n'en a jamais tiré les conséquences qui s'imposaient vis-à-vis de sa narratologie. Dès lors que l'on considère le paratexte comme un acte promissif, la sincérité de cet acte dépend de la confiance que l'on accorde à celui qui l'accomplit. L'auteur n'est plus forcément une garantie, une instance "reliable", digne de confiance, comme diraient les Américains à propos du narrateur, en sorte que les égalités ou inégalités dont je viens de parler $(\mathrm{A}=\mathrm{N}$ ou $\mathrm{A} \neq \mathrm{N})$ n'ont plus le statut de preuve indubitable. De plus, l'auteur devient un acteur parmi d'autres de la communication, l'éditeur prenant une place prépondérante.

De ce déplacement de paradigme découlent l'abandon de l'idée de contrat et l'alignement de la communication médiatique sur le modèle publicitaire, dans lequel, on le sait, la promesse est un fondement argumentatif: on pourrait appliquer cette formule d'Adam et Bonhomme à propos du discours publicitaire : elle a un " être monologique » et un "paraître dialogique » (1997, p. 37).

Tout cela m'amène à une première conclusion provisoire : ce qui caractérise le récit médiatique, ce n'est pas un simple déplacement de normes par rapport au récit en général, comme le laisse accroire la façon dont Genette traite les différences entre récit de fiction et récit de réalité. C’est bien plus : le récit médiatique est de l'ordre de la persuasion : il ne s'adresse pas à des lecteurs ou à des spectateurs conçus comme de purs êtres de raison, cultivés, lecteurs de narratologie et déjà 
convaincus de la position à adopter pour lire ou voir un bien culturel, mais à des téléspectateurs qu'il faut persuader du statut de l'objet.

\section{De l'image aux genres}

De tout ce qui précède on peut aussi déduire qu'il ne saurait y avoir de sémiotique des médias unifiée. Bien que l'expression circule dans l'espace universitaire, au point qu'on recrute parfois des enseignants sous cet intitulé, on voit mal comment on pourrait traiter pareillement la presse écrite, qui articule le texte verbal et l'image fixe, la télévision et ses cinq matières de l'expression ou la radio qui est fondée sur l'oralité. Les sémiologies de l'écrit, de l'image fixe et de l'image animée ne forment pas un corps de concepts unifié. Comment dans ces conditions imaginer que les médias ressortissent à une sémiotique unique? Certes, tous ont en commun quelques mécanismes et un fonctionnement fondé sur la promesse, mais, pour aller plus avant dans la mise au jour de celui-ci, il est nécessaire de prendre en compte la matérialité des "médiums » propres au média concerné. On n’étudie pas la maquette d'un journal comme une émission de télé-réalité. Aussi, cet article ne prétend-il nullement décrire ce que fait la sémiologie à l'analyse des médias en général, mais seulement rendre compte des modifications de paradigme requises par l'étude de la télévision.

Venant moi-même de la sémiologie du cinéma, je me souviens comme je fus dérouté quand il me fallut trouver des outils qui me permettraient d'explorer les dizaines de milliers d'heures de télévision que mettait à la disposition du chercheur l'Inathèque de France. Face à des images de documentaires, de magazines, de téléfilms, de journal télévisé, de météo, etc., à quoi allait me servir tout ce que je savais de l'image cinématographique et de son équivalent, à la différence de taille près, l'image télévisuelle? Quand j'aurais dit que la présentatrice de la météo est en plan moyen, que les émissions littéraires ne mettent en œuvre aucune des configurations de la "grande syntagmatique de la bande-image», qu'aurai-je compris de la télévision et qu'en aurai-je fait comprendre? Pas grand-chose. C'est en comparant la narration simultanée au cinéma et le direct télévisuel que j'en acquis la conviction (Jost 1998). En l'occurrence, je rapprochai deux séquences. L'étonnante scène d'E la nave va, dans laquelle Orlando commente ce qu'il voit comme un véritable reporter:

Le grand-duc est suivi de sa sœur Lerimia, la princesse aveugle qui a perdu la vue tout enfant. Pourtant, vous avez remarqué, elle marche à côté du Premier ministre avec une incroyable assurance...

Et la retransmission en direct des obsèques du roi Baudouin décrite par L. Zitrone :

Retour sur les majestés... Juan Carlos, Sophie, Béatrix, roi de Suède, la reine de Suède, née Sommerlatt, qui a connu son mari dans des circonstances extraordinaires. Elle était employée... 
En termes narratologiques, ces deux séquences sont strictement équivalentes : elles sont toutes deux en narration simultanée, la direction d'ajustement du mot aux images est vectorisée par l'observation du monde et elles décrivent la réalité sous l'aspect imperfectif: l'action est en train de s'accomplir devant nos yeux. Rien ne distingue ces deux séquences du point de vue du fonctionnement textuel, et pourtant le spectateur les interprète différemment grâce à ce que Schaeffer (1987) a appelé, s'agissant de la photo, le savoir de l'arché. En l'occurrence, ce savoir trace une frontière entre la classe des empreintes par connexion physique produites dans le passé, le second à celle des empreintes par connexion physique produites dans le présent. Les deux séquences se distinguent l'une de l'autre grâce aux inférences du spectateur à partir de ses savoirs sur l'image. Dans cette perspective, les travaux de Sperber et Wilson, qui s'en prenaient vivement à la notion de code dans $L a$ Pertinence (Sperber et Wilson 1986), laissaient entrevoir des avancées fructueuses, en mettant en lumière que la communication verbale était largement fondée sur des inférences des acteurs et sur leur partage d'un univers cognitif commun.

La fonction cognitive du genre, pour le téléspectateur, pouvant être définie de façon générale, comme une recherche de pertinence, c'est-à-dire comme un effort pour augmenter sa connaissance du monde, il est naturel de se demander dans un premier temps comment le spectateur fait pour ramener l'inconnu au connu, en d'autres termes, quelles inférences il met en œuvre à partir de la réalité textuelle. Je fais donc l'hypothèse, dans Un monde à notre image (Jost 1992), que la catégorisation des documents dépend des inférences opérées par le spectateur à partir de ce document sur l'intention qui le sous-tend.

À cet égard, le spectacle télévisuel du procès de Ceausescu, en 1989, fut pour moi une expérience déterminante puisque, par un hasard des calendriers, elle intervint en plein débat narratologique : le document montrait le couple de dictateurs face à des juges toujours invisibles. À espaces réguliers, l'image se figeait. En bon narratologue, dans la mesure où «l'on ne saurait les considérer [l'arrêt sur image ou le plan immobile] comme des énoncés narratifs » (Gaudreault 1988, p. 47), on aurait dû considérer ces frozen shots comme des arrêts du récit; en bon technicien, on aurait pu penser aussi qu'il s'agissait d'un problème technique de ruptures de faisceau. Or, comme le confirma le document complet quelques jours plus tard, ces figés étaient des coupes volontaires des juges, c'est-à-dire des actes de récit, identifiés ici à de la censure, qui intervenaient à chaque fois que la caméra s'était tournée vers eux et les avaient montrés dans cette parodie de procès.

D'un exemple comme celui-ci, et de bien d'autres naturellement, on peut conclure que le spectateur formule, parfois en cours de projection, parfois avant, une ou plusieurs hypothèses sur la nature du programme, notamment sur le degré d'intervention humaine dans l'élaboration du document et sur sa finalité. Ainsi n'attribue-t-on pas le même sens à des images d'un procès, selon qu'elles proviennent de caméras de surveillance, d'un montage a posteriori ou d'une caméra cachée. L'articulation de ces hypothèses entraîne le spectateur à recevoir 
un document comme une promesse ontologique, c'est-à-dire une promesse consubstantielle de l'horizon d'attente du genre, qui le guide dans sa perception : promesse sur la relation liant l'image et la réalité (authenticité); promesse sur le niveau pertinent d'analyse ou de synthèse des images et des sons pour la compréhension narrative ou esthétique ${ }^{4}$. Le direct non préparé est lié à une temporalité subie, dont la retransmission tente de rendre compte avec les moyens du bord (sans avoir pouvoir décider des emplacements de caméra, par exemple) et s'accompagne d'une promesse d'authenticité. En revanche, le documentaire construit l'espace et le temps en fonction de la réalité et est fondé sur une promesse de lisibilité accrue du réel. Quant à la fiction, sa construction spatio-temporelle est déterminée par la communication de l'intrigue, s'appuyant sur une promesse de pertinence narrative du visible et de l'audible. Ces résultats, que je résume à grands traits au risque de les dénaturer (mais il faut vivre dangereusement!) ont trouvé un terrain d'élection avec l'arrivée de la télé-réalité. Quand, au lancement de Loft story, un cinéaste comme Beineix s'émerveille du naturel des candidats, capables de dialogue d'une vérité criante que le cinéma ne serait plus capable d'atteindre, il reste prisonnier de la logique immanentiste qui est celle de la première sémiologie audiovisuelle, logique qui est incapable de formuler la différence entre un film de fiction et une retransmission en quasi directe. Malgré les ressemblances de contenu de la longue séquence de Jeanne Dielman 23 quai du commerce 1080 Bruxelles, au cours de laquelle Delphine Seyrig épluche des pommes de terre, et une séquence de préparation de repas dans le loft, le fait que dans la première le téléspectateur sait que la scène est là pour signifier, dans la mesure où elle est fictionnelle, alors que dans la seconde elle ne fait que montrer ce qui se passe dans l'appartement des candidats, les rend profondément différentes.

\section{Que signifie « communication " pour les médias?}

Parvenu en ce point, on pourrait critiquer les propositions précédentes, en objectant que ce que j'appelle "promesse » ressemble bigrement à ce que d'autres appellent « contrat» ou « horizon d'attente». En fait, la ressemblance n'est qu'apparente. En premier lieu, le contrat suppose que la connaissance des genres est universelle et partagée. Par exemple, que tout un chacun s'accorde sur le « contrat de lecture » de la fiction qui commande la suspension of disbelief. Or, comme je l'ai souvent répété, il s'en faut de beaucoup. Je renvoie au constat d'Eco, après la sortie du Pendule de Foucault, devant les nombreux courriers qui contestent tel ou tel détail de ses romans en s'appuyant sur leur connaissance de la réalité :

Je me suis rendu compte d'un phénomène extraordinaire. Jusqu'à quelques dizaines de milliers d'exemplaires (estimation variable d'un pays à l'autre), on touche en général un public connaissant parfaitement le pacte

4. Je développe cette idée dans Jost (1997). 
fictionnel. Après, et surtout au-delà du million d'exemplaires, on entre dans un no man's land où il n'est pas sûr que les lecteurs soient au courant de ce pacte (Eco 1994, p. 102 tr. fr.).

Autrement dit, il n'est pas donnéà tout le monde de savoir ce qu'il faut attendre d'une fiction. La connaissance de ce que peut promettre une fiction, un documentaire ou un direct nécessite donc à la fois des recherches fondamentales et l'apprentissage des savoirs qu'elles ont formalisés. D’où la nécessité d'une éducation aux médias.

En deuxième lieu, même si on est « au courant » de ce pacte, la télévision a ceci de particulier qu'elle est un flot ininterrompu d'images, de paroles et de sons, dans lequel le téléspectateur entre souvent par hasard, soit qu'il l'allume sans connaître les programmes, soit qu'il découvre une émission au détour d'un zapping. Dès lors, il lui faut parfois un bon moment pour identifier le genre auquel appartient ce qu'il regarde. Cette difficulté est évidemment hors du champ d'interrogation de la sémiologie immanentiste qui suppose qu'on entre dans un texte dans l'ordre chronologique de la «bonne» lecture : depuis la couverture jusqu'à la dernière page en passant par les pages de titre.

En troisième lieu, en raison de sa nature médiatique, qui ne saurait se décrire, on l'a vu, dans les termes d'un circuit de communication où codage et décodage sont deux opérations symétriques et transitives, le programme fait l'objet d'une promesse, qui s'ajoute et module la promesse inhérente à chaque genre. Les différents éléments, écrits, oraux ou audiovisuels qui accompagnent le lancement d'un programme ne sont plus considérés dans ces conditions comme des « seuils » ou des «consignes" pour (bien) l'interpréter mais comme des actes promissifs qui n'engagent que l'émetteur (producteur et/ou diffuseur) et que le téléspectateur peut croire ou non en fonction de la confiance qu'il lui accorde et de ses propres connaissances sur les genres (et leur promesse ontologique). Cette promesse pragmatique passe par les dossiers de presse, la promotion d'un programme par ses producteurs, la publicité, les bandes-annonces, etc.

Pour que ce système communicationnel fonctionne encore faut-il qu'émetteurs et récepteurs se rencontrent sur un terrain commun. Ce terrain ne peut être l'image, trop étroit pour permettre l'interprétation globale d'un programme. La promesse pragmatique qui accompagne tout programme suggère que la monnaie d'échange ayant cours dans la communication médiatique est le genre. Pour comprendre sa fonction, la démarche de Schaeffer (1989) consistant à corréler les étiquettes génériques à ce qu'elles disent de leur fonction communicationnelle est séduisante. Elle permet notamment de rendre compte de la création indéfinie de ces étiquettes, particulièrement vive pour les productions télévisuelles. Néanmoins elle a, pour moi, deux défauts : elle donne un rôle exagéré à l'onomaturge, qu'il soit producteur ou diffuseur et, ce faisant, elle aboutit à l'inverse de ce à quoi elle voulait parvenir : tout en visant une perspective communicationnelle, elle fige les genres en une répartition statique, qui serait valable pour n'importe qui, quel que soit l'usage que l'on fait du genre. Or les noms de genres ne sont pas des entités sémantiques 
stables, leur interprétation varie en fonction des acteurs de la communication. Un diffuseur peut fort bien présenter un film comme La Rafle comme un documentaire sur la rafle du Vel d'Hiv' et le téléspectateur le regarder comme une fiction; annoncer Secret Story comme une "télé-réalité », alors que d'aucuns le regardent comme un pur divertissement. Si l'on peut toujours décrire les traits pertinents qui caractérisent un genre, la communication médiatique procède par méta-catégorie qui rattache chaque genre à un archi-genre, qui en est l'interprétant. Selon les cas, cet interprétant est une proposition, une promesse ou une injonction de sens. Soit le programme renvoie à notre monde, soit il renvoie à un objet mental (la fiction), soit il se prend lui-même comme objet, ce qui est toujours le cas du jeu, qui fonctionne en partie en circuit fermé et sous forme réflexive, obéissant aux règles qu'il s'est fixées. Cette tripartition m’a amené à considérer la communication télévisuelle comme un terrain délimité par un triangle dont les trois angles sont le monde réel, le monde fictif et le monde ludique, sur lequel l'emplacement d'un genre dépend en partie des acteurs de cette communication. Grâce à ce modèle théorique, j'ai ainsi pu montrer comment le procès dont l'île de la tentation ${ }^{5}$ a fait l'objet ces dernières années a vu s'affronter trois argumentations qui, chacune tirait le programme vers un de ces mondes : la promesse pragmatique en faisait une expérience réelle, au cours de laquelle des couples éprouvaient la solidité de leur amour; le producteur plaidait qu'il ne s'agissait que d'un divertissement et d'activités ludiques, tandis que les avocats des candidats démontraient que leur statut était proche de celui des artistes de spectacles. Mon analyse du programme allait plus loin encore en l'assimilant à un spectacle d'acteurs de ligue d'improvisation.

Ce dernier rapprochement, entre la lutte pour l'interprétation générique des acteurs de la communication et mon analyse, ainsi que tout ce qui précède, nous ramène à mon paradoxe de départ et permet d'y apporter une première réponse. Je ne suis pas sémiologue si l'on entend par sémiologie tout ce que je viens de critiquer, mais je le reste si l'on admet que la compréhension d'un processus communicationnel mis en œuvre par un média rend nécessaires une prise en compte du contexte au sens large et l'aide de l'histoire et des conditions socio-économiques de sa production. Une véritable approche pragmatique ne doit pas se contenter d'élargir l'empan textuel pour comprendre comment se règle l'interprétation. Elle doit accepter ce fait que la communication n'est pas un long fleuve tranquille et que la coopération que suppose le modèle du contrat est une fiction optimiste qui imagine les relations entre ses acteurs comme pacifiques. Les nombreux procès qu'a entraînés la création de programmes de télé-réalité rappellent à qui veut l'entendre que le sens ne se construit pas toujours avec, qu'il se construit aussi contre. Dire cela, ce n'est pour autant tomber dans un relativisme dont le mot d'ordre serait «à chacun sa vérité ». Soutenir que l'île de la tentation est une " télé-réalité », un divertissement ou une fiction ne revient pas au même. Tout le monde n'a pas

5. Des candidats ont fait un procès au producteur pour faire reconnaître leur activité comme un travail. Les premiers procès ont eu lieu en 2005. Il y a plus d'une centaine de plaignants. 
raison. Si l'on sait ce que fiction veut dire, on doit bien admettre que ce programme en est proche. Mais, pour arriver à cette conclusion, il ne suffit pas d'analyser le texte que constituerait le programme ou un corpus de plusieurs programmes. Il faut aussi connaître les conditions de production : savoir, par exemple, qu'on suggère aux candidats des situations, qu'on leur fait répéter des répliques, qu'on les " coache » quand ils ne sont pas assez convaincants, etc. Tous ces savoirs imposent évidemment d'aller au-delà d'une théorie immanentiste. Cependant, il faut aussi déterminer ce qu'on peut entendre par fiction s'agissant d'images animées, ce qui est loin d'aller de soi; définir ce qu'est un jeu, s'interroger sur le type de réalité que construit l'image, etc. : autant de thèmes de recherche qui relèvent bien d'une interrogation sémiologique et philosophique.

Dès lors, cette interrogation est un moyen terme entre les promesses de l'émetteur, qui peut bien promettre n'importe quoi, et les diverses interprétations que suscite un programme. Quand un producteur promeut son docu-fiction sur la préhistoire en affirmant qu'on peut le regarder comme un documentaire sur cette époque, plus fiable qu'une leçon d'histoire, le sémiologue peut objecter qu'un documentaire filme un état du monde qui lui préexiste, un monde afilmique, et non un monde profilmique construit pour la caméra et joué par des acteurs. Quand des téléspectateurs prennent pour argent comptant des scènes d'intimité jouées par une famille, il est bon de rappeler qu'il ne s'agit pas d'une scène prise sur le vif, mais d'une scène feinte, d'une feintise. La mise au jour des caractères définitoires des genres et des interprétants que sont les mondes est donc un étalon nécessaire pour juger les promesses pragmatiques et comprendre la réception. Et ces trois moments ont ceci en commun de se dérouler sur le même terrain.

\section{Du signe au symptôme}

On se souvient peut-être que les Mythologies de Barthes avaient une double ambition : une "critique idéologique portant sur le langage de la culture dite de masse » et «le démontage sémiologique de ce langage ». Au fil des ans, la première a fini par se diluer et la sémiologie par former un territoire autonome déconnecté de l'analyse de la société. Or, si l'analyse des programmes et de la programmation télévisuels supposent une prise en compte du contexte au sens large, il me semble que, par un juste retour des choses, le devoir du théoricien des médias est d'articuler leur signification à la société dans laquelle ils se développent. Cela ne veut pas dire qu'il faut délaisser la sémiologie au profit de la sociologie, comme le croient certains sociologues qui voient en Barthes un des leurs, aveugles sur son travail de « démontage sémiologique du langage ", mais plutôt qu'il faut s'en servir pour comprendre notre société. Il ne suffit pas de montrer comment se produit le sens, il faut aussi interroger sur son pourquoi. Pourquoi la télé-réalité a-t-elle connu le succès que l'on sait alors que ses nombreux prodromes dans les deux dernières décennies du $\mathrm{xx}^{\mathrm{e}}$ siècle n'en ont pas eu? Pourquoi les séries américaines, méprisées 
naguère, suscitent-elles aujourd'hui autant d'addictions aujourd'hui ? Et pourquoi certaines sont-elles plus populaires que d'autres? Ces questions auxquelles deux de mes ouvrages récents se sont efforcés d'apporter des réponses (Jost 2009 et 2011) amènent à sortir les relations du programme à la société de l'alternative dans laquelle on les pense habituellement.

Le cadre de pensée le plus ancien et le plus rabâché est celui fourni par Adorno et Horkheimer : il confère aux médias un rôle déterminant dans la fabrication de nos visions du monde, et soutient que notre imaginaire est largement tributaire des idées ou des émotions que leurs programmes construisent. Le second, en vogue chez les tenants des Cultural studies, voit dans les contenus des programmes, le reflet de nos sociétés, de leurs structures ou de leurs contradictions. La première thèse est quasi-impossible à prouver et la seconde peine à aller au-delà des évidences : une fois que l'on a dit que le processus d'élimination des candidats est à l'image de notre société d'exclusion, on n'est guère plus avancé.

Je préfère donc envisager le programme sous un troisième angle redonnant à la sémiologie ou à la séméiologie le sens qu'elle a en médecine : l'étude des symptômes. De même que le mythe chez Barthes ne se donne jamais sous le signe de l'évidence, le symptôme n'est pas, contrairement au reflet, un signe transparent dont la signification apparaîtrait au seul regard. Il ne ressemble pas non plus forcément à l'objet dont il renvoie ou déforme l'image. Plutôt qu'une icône, il est un indice qui garde la trace de son objet sans forcément lui ressembler : un mal de tête ne ressemble pas à sa cause, mais y renvoie. Le symptôme est aussi plus qu'un signe : c'est un signe de crise ou, tout au moins, d'un fonctionnement pathologique du corps. Considérer les programmes comme des symptômes, c'est donc à la fois les considérer comme signes d'un objet qu'ils ne manifestent pas explicitement, mais par une signification latente, et envisager dans quelle mesure ils disent quelque chose d'un état de la société, de la politique ou des téléspectateurs, ce "quelque chose » étant dans certains cas un dysfonctionnement, un malaise ou la part sombre de nous-mêmes.

Pour exemplifier cette relation du programme à la société dans laquelle il se développe, je me contenterai ici d'un exemple, que j'ai développé dans Télé-réalité. En 2001 débarque en France Loft Story, adaptation du format international Big Brother. Le programme connaît très rapidement un gros succès. Quels sont les traits saillants de ce format, que les producteurs ont baptisé " télé-réalité » au lieu de se contenter de traduire l'étiquette générique d'origine : "real-life docusoap"? D’abord, bien sûr, le fait qu'il prétend avoir comme interprétant principal la réalité. Et ce pour deux raisons: d'une part, parce que les candidats sont des « vraies " gens; d'autre part, parce que le téléspectateur va pouvoir observer leur vie en direct, et que la promesse ontologique, on l'a vu, est l'authenticité. En fait, ces deux promesses pragmatiques sont très contestables : les participants sont « castés » en fonction de leurs virtualités narratives, la retransmission n'est pas en direct, mais en très léger différé. Toutefois, à l'époque, elles ne sont guère remises en cause par le public. 
Pourquoi a-t-il envie de croire à la réalité de ce programme? Pour le comprendre, il faut revenir au contexte socio-historique dans lequel il intervient. Durant la décennie qui a précédé Loft Story, la confiance des citoyens dans leurs médias n'a cessé de s'éroder. En 1989, l'affaire du faux charnier de Timisoara, survenue lors de la chute de Ceausescu, a brusquement déplacé la nature de la sémiose proposée par les médias : alors que les téléspectateurs étaient habitués à considérer la télévision comme une fenêtre sur le monde et, ce faisant, à renvoyer les images à ce qu'elles représentaient, beaucoup ont pris conscience que l'objet des images pouvait être celui ou ceux qui les avaient fabriqués et diffusés. D’une sémiologie de l'énoncé, on a glissé brusquement à une sémiologie de l'énonciation. Deux ans plus tard, ce sentiment de manipulation s'est renforcé avec les retransmissions de la première guerre du Golfe : l'impression de ne pas avoir vu à la télévision des images qui en rendaient compte fut clamée haut et fort par Jean Baudrillard, qui, malgré ce que l'on savait des atrocités commises, put écrire La Guerre du Golfe n'a pas eu lieu. Ces événements et d'autres encore aboutissent en 2000 au fait que la confiance des Français en leurs médias chute, en même temps que leur crédibilité, de $58 \%$ à $47 \%{ }^{6}$. Ce sont les jeunes qui sont les plus critiques et qui expriment une insatisfaction sur la façon dont sont traités certains événements. Dans ces conditions, la promesse d'authenticité du direct de Loft story apparaît comme un bol d'air et un antidote à la manipulation médiatique (cela changera dans la décennie suivante).

Une autre promesse pragmatique accompagne la diffusion de Loft Story: le rôle dévolu aux téléspectateurs. "Cette histoire, c'est vous qui allez l'écrire ", lance le présentateur à l'adresse du public le premier soir. C'est effectivement la vraie nouveauté de ce type de programme : les téléspectateurs vont voter par SMS surtaxés pour éliminer les candidats qui leur déplaisent. Le téléphone portable est alors en pleine expansion et les jeunes appellent en masse. Un an plus tard, en mai 2002, 37\% des 18-24 ans s'abstiennent au premier tour des élections présidentielles ${ }^{7}$, qui qualifie Le Pen pour le second tour. La soif de voter dont a témoigné le lancement du Loft contraste avec la participation à une élection dont l'importance est réelle. De quoi cet écart est-il le symptôme? Le rapprochement avec des enquêtes effectuées la même année et des analyses de politologues nous éclairent. En 2001, 75\% des Français déclarent ne pas faire confiance aux hommes politiques, alors qu'ils n'étaient que $55 \%$ en $1985^{\circ}$ et ils ne sont que $30 \%$, dans la même enquête, à faire confiance aux médias. Là encore, les jeunes sont les plus sévères : $84 \%$ des 18-24 ans mettent ainsi en doute l'honnêteté des hommes politiques contre seulement $52 \%$ des plus de 65 ans $^{9}$. Pour autant, cette méfiance envers ceux qui nous gouvernent ne s'accompagne pas d'un dégoût du

6. Sondage La Croix-Télérama réalisé entre le 20 et 26 décembre 2000 par la SOFRES.

7. Sondage Ipsos, source le Monde, 25 avril 2002.

8. Sondage du SOFRES du 27 et 28 juin 2001. Ces données sont tirées de l'article de Balme et al. (2003).

9. Le Monde, $17 / 4 / 2002$. 
vote. Une étude montre que les Français regrettent surtout l'« hiatus entre l'offre électorale et [les] attentes ${ }^{10}$. Ils ne se sentent pas bien représentés et c'est ce défaut de représentation qui entraîne la défiance envers la politique. On reproche aux hommes politiques d'être insincères, seulement soucieux de plaire aux électeurs, d'appartenir à un autre monde, celui des puissants et des nantis - « ils ne sont pas comme nous " - et, enfin, de méconnaître le quotidien des citoyens ${ }^{11}$.

Dans ces conditions, le succès de Loft Story apparaît bien comme le symptôme de la défiance politique, qui est elle-même le "signe d'une crise » ${ }^{12}$. Les promesses pragmatiques décrites supra répondent bien mieux que les promesses électorales aux défauts imputés aux politiques : d'abord, en mettant en scène, des gens sans qualités particulières, dont on fait croire à l'époque qu'ils sont représentatifs des Français; ensuite, en leur demandant d'être eux-mêmes et en exigeant d'eux la sincérité ("Soyez-vous même!» est le slogan de l'émission); enfin, en faisant dépendre leur sort du vote des téléspectateurs. Ces trois promesses prises à la lettre expliquent que les soirées de prime time pendant lesquelles on procède à l'élection des sortants fassent les plus grosses audiences. Elles comblent le besoin de voter tout en offrant la possibilité de recadrer la relation représentationnelle des candidats avec les téléspectateurs.

Loft Story peut donc être compris comme un symptôme à double titre. D'une part, comme symptôme d'un phénomène social beaucoup plus large et qui a pris de l'ampleur dans les années précédentes : la méfiance envers les médias, que le programme viendrait en quelque sorte rémunérer. D'autre part, comme prodrome, comme signe avant-coureur d'un événement politique historique : le désintérêt des jeunes pour l'élection du président de la République, qui va fortement peser sur les résultats de la présidentielle de 2002.

Contrairement au texte en général, qui peut être étudié pour lui-même, abstraction faite de tout ancrage contextuel, l'objet médiatique ne prend son sens que dans et par la communication. On peut certes traiter une série télévisuelle comme un texte et montrer l'ingéniosité de son fonctionnement, la façon dont sont construits les personnages ou dont elle invente la temporalité. Pour légitime qu'elle soit, une telle attitude ne met au jour que la dimension audiovisuelle du programme, ne le traitant pas différemment d'un film. Ce n'est d'ailleurs pas un hasard si beaucoup d'analystes du cinéma sont passés à l'étude des séries. Étudier ces objets comme des programmes télévisuels, c'est-à-dire comme des objets médiatiques, est une autre démarche qui implique une autre méthode. Il faut comprendre comment ils s'articulent aux autres programmes (par exemple, quelles relations ils entretiennent avec la télé-réalité), comment ils sont programmés et quel rôle joue la programmation dans leur succès mais, surtout, de comprendre

10. Balme et al. (2003, p. 445).

11. Ibid. (p. 446).

12. Ibid. (p. 460). 
ce que ce succès ou ces échecs disent de la société qui est leur terreau et de nousmêmes. La séméiologie des médias n’en est qu’à ses débuts.

\section{Références bibliographiques}

Adam, Jean-Michel et Bолноммe, Marc (1997), L'Argumentation publicitaire, Paris, Nathan universitaire.

BALme, Richard et al. (2003), "Les motifs de la confiance (et de la défiance) politique : intérêt, connaissance et conviction dans les formes du raisonnement politique ", Revue Internationale de Politique Comparée, vol. 10, $\mathrm{n}^{\circ} 3$.

Delavaud, Gilles (2005), L'Art de la télévision, Bruxelles-Paris, De Boeck-INA.

Eco, Umberto (1994), Six Walks in the Fictional Woods, Harvard University Press; tr. fr. Six promenades dans les bois du roman et ailleurs, Paris, Grasset.

Gaudreault, André (1988), Du littéraire au filmique. Système du récit, Paris, Méridiens Klincksieck.

Genette, Gérard (1972), Figures III, Paris, Seuil, coll. Poétique.

— (1987), Seuils, Paris, Seuil, coll. Poétique.

Jost, François (1992), Un monde à notre image. Énonciation, cinéma, télévision, Paris, Méridiens Klincksieck.

— (1997), «La Promesse des genres », Réseaux, vol. 15, n. 81, pp. 11-31.

- (1998), Le Temps d'un regard. Du spectateur aux images, Québec-Paris, Nota BeneKlincksieck.

- (2009), Grandeurs et misères de la télé-réalité, Paris, Cavalier bleu, coll. Mythologie.

- (2011), De quoi les séries américaines sont-elles le symptôme?, Paris, CNRS éditions, coll. Débats.

Lejeune, Philippe (1975), Le Pacte autobiographique, Paris, Seuil, coll. Poétique.

Metz, Christian (1968), Essais sur la signification au cinéma, Paris, Klincksieck.

- (1971), Langage et cinéma, Paris, Larousse, coll. « Langue et Langage».

Schaeffer, Jean-Marie (1987), L'Image précaire, Paris, Seuil, coll. Poétique.

- (1989), Qu'est-ce qu'un genre littéraire?, Paris, Seuil, coll. Poétique.

Souriau, Étienne (éd., 1953), L'Univers filmique, Paris, Flammarion.

Sperber, Dan et Wilson, Deirdre (1986), Relevance. Communication and Cognition, Harvard University Press; tr. fr. La Pertinence. Communication et cognition, Paris, Minuit, 1989.

Véron, Eliseo (1983), « Il est là, je le vois, il me parle », Communications, 38, pp. 98-120. 